\title{
A New Approach to Image Fusion Based on Cokriging
}

\author{
Nargess Memarsadeghi \\ NASA/GSFC \\ Greenbelt, MD 20771 \& \\ University of Maryland \\ nargess@cs.umd.edu
}

\author{
Jacqueline Le Moigne \\ NASA/GSFC \\ Greenbelt, MD 20771 \\ lemoigne@backserv.gsfc.nasa.gov
}

\author{
David M. Mount* \\ University of Maryland \\ College Park, MD 20742 \\ mount@cs.umd.edu
}

\author{
Jeffrey T. Morisette \\ NASA/GSFC \\ Greenbelt, MD 20771 \\ Jeff.Morisette@nasa.gov
}

\begin{abstract}
We consider the image fusion problem involving remotely sensed data. We introduce cokriging as a method to perform fusion. We investigate the advantages of fusing Hyperion with ALI. This evaluation is performed by comparing the classification of the fused data with that of input images and by calculating well-chosen quantitative fusion quality metrics. We consider the Invasive Species Forecasting System (ISFS) project as our fusion application. The fusion of ALI with Hyperion data is studied using PCA and wavelet-based fusion: We then propose utilizing a geostatistical based interpolation method called cokriging as a new approach for image fusion.
\end{abstract}

Index Terms-Image Fusion, Cokriging, Wavelet, PCA, Remote Sensing, Hyperion, ALI

\section{INTRODUCTION}

In the remote sensing domain, image fusion is a technique which deals with the limitations of sensors in capturing high spectral/spatial resolution multispectral images [1]. In this paper, we study fusion of two remotely sensed data sets (ALI and Hyperion) using PCA and wavelet-based fusion. We also propose a new image fusion approach based on cokriging [2]-[4]: Cokriging is an interpolation method for a variable available at scattered data points using multiple variable values of different natures. at nearby locations, and thus we found it suitable in addressing data/image fusion needs. We perform preliminary experiments for fusion based on cokriging using our given data sets as a preliminary proof of concept experiment.

The goal of our project is to eventually evaluate our fusion results by performing classification of the fused data and by measuring the classification accuracy using ground truth from the ISFS application. In the absence of ground truth we consider other quantitative quality metrics. In this paper, we study the advantages of fusing Hyperion bands with their corresponding ALI bands by studying the amount of details gained. We also mention problems that need to be addressed when designing future fusion metrics.

In Section II we give an overview and definition for data and image fusion. Then, in Section III we give an overview of our application project, ISFS, and its objectives. We describe the data sets used for our experiments in Section IV. In

\footnotetext{
*The work of this author was supported by the Science Foundation under grant CCR-0098151.
}

Sections V and VI the fusion methods used in our experiments are described. We mention evaluation methods we considered for our experiments' results in Section VII and present the results in Section VIII. After explaining the advantages and limitations of the used fusion methods, we give an overview of the cokriging interpolation method in Section V-C, propose it as a new approach for data fusion, and perform preliminary tests on our data set. Finally, we give conclusions and overview of our future work in Section IX.

\section{DATA AND IMAGE FUSION}

Data fusion was originally defined as a group of methods and approaches using multi-source data of different natures to increase the quality of information contained in the data [5]. This and other similar definitions were considered rather restrictive later. More recent definition of data fusion describes it as "a process dealing with the association, correlation, and combination of data and information from single and multiple sources to achieve refined position and identity estimates, and complete and timely assessments of situations and threats, and their significance" [6]. Thus, while the original definition conveys the idea of the combination of data, the new definition also considers the study of correlation among data of different sources to generate and evaluate hypothesized associations among data [6], and in fact this is the definition we are considering for data fusion in this paper by studying the relationship between ALI and Hyperion data through image fusion.

Image fusion refers to data fusion where the data used are images of multiple sources. There are many objectives of image fusion including image sharpening, improving registration/classification accuracy, temporal change detection, feature enhancements, etc; [5]. In this paper, our objective for image fusion is the improvement of classification accuracy for our target application (ISFS, see Section III) as well as feature enhancement which can lead to better classification.

\section{ISFS: INVASIVE SPECIES FORECASTING SYSTEM}

Our underlying application involves the analysis of invasive species through a collaborative project among. NASA Office of Earth Science and the US Geological Survey called Invasive Species Forecasting System (ISFS) [7], [8]. An invasive 
species is defined as any non-native species whose introduction causes or is likely to cause harm to the economy, environment, or to human health. The economic damages caused by invasive species to agricultural producers and commercial fishery alone run to billions of dollars each year [8]. Tamarisk, Russian olive, leafy spurge, and water hyacinth are examples of various invasive species under study. The data sets used in this paper's experiments are from one of the four main Tamarisk study sites here in Colorado.

While many vegetation types may appear to have the same color when viewed in the visible spectrum, they can be differentiated from each other when viewed in the infrared or ultra violet spectra [9]. Even when viewed in the non-visible spectrum, reflectance of these vegetation types may be of different degrees and from nearby portions of the spectrum. For this reason hyperspectral data is of great importance to the ISFS project. However, one would need to choose its appropriate bands for a particular study based on the application. In this paper we try to investigate candidate choices for Hyperion bands to be used for the ISFS project by learning the amount of detail that they introduce to the classification compared to their corresponding ALI bands.

\section{Data Sets}

Our data sets were acquired in July 5, 2004 from "Debeque" (near Grand Junction, Colorado, U.S.A) site which is one of the four study sites for ISFS's Tamarisk mapping effort [7]. The Advanced Land Imager (ALI) and Hyperion are two instruments on the Earth Observing 1 (EO-1) platform. Hyperion is a hyperspectral instrument with 242 bands covering wavelengths ranging from $356 \mathrm{~nm}$ to $2577 \mathrm{~nm}$ at a spatial resolution of 30 meters per pixel. ALI on the other hand, has only 10 bands, one of which is panchromatic at 10 meters spatial resolution and 9 of which are multispectral at a 30 meters spatial resolution, covering wavelengths ranging from $433 \mathrm{~nm}$ to $2350 \mathrm{~nm}$. Thus, ALI data represent low spectral resolution data while Hyperion provides high spectral resolution images. ALI is considered a successor system to the Landsat Thematic Mapper series, and thus 5 of its multispectral bands' wavelengths correspond to that of Landsat 7. Hyperion is the only civilian hyperspectral instrument operating in space.

For this paper, we used two data sets one obtained from ALI and one from Hyperion instrument, containing approximately the same area. We had the 9 multispectral bands of ALI as well as all Hyperion bands for the region under study.

Our objective of performing various image fusion techniques on two images, one with low spectral resolution and one with high spectral resolution, is to study how much we can improve the quality of the classification performed on a Landsat/Landsat type image using hyperspectral data. Having both ALI and Hyperion on the same platform reduces temporal effects on registration accuracy greatly.

The ALI data were georeferenced, while the Hyperion data did not have any associated map information. Thus, we registered Hyperion data to ALI, using ALI as our reference image. We performed registration in a two step process:
TABLE I

ALI AND MATCHING CALIBRATED AND NOT CORRUPTED HYPERION BANDS USED (CWL = CENTRAL WAVELENGTH)

\begin{tabular}{|c|c|c|c|c|}
\hline $\begin{array}{c}\text { ALI } \\
\text { MS } \\
\text { Bands }\end{array}$ & $\begin{array}{c}\text { Spectral } \\
\text { Range } \\
(\mathrm{nm})\end{array}$ & $\begin{array}{l}\text { CWL } \\
(\mathrm{nm})\end{array}$ & $\begin{array}{c}\text { Matching } \\
\text { Used } \\
\text { Hyperion } \\
\text { Bands }\end{array}$ & $\begin{array}{l}\text { CWL } \\
\text { (nm) }\end{array}$ \\
\hline $\begin{array}{c}1 \\
\left(M^{\prime}-1^{\prime}\right)\end{array}$ & $433-453$ & 441.6 & $\frac{9}{10}$ & $\begin{array}{l}436.99 \\
447.17\end{array}$ \\
\hline $\begin{array}{c}2 \\
(\mathrm{MS}-1)\end{array}$ & $450-515$ & 484.8 & $\begin{array}{l}11 \\
\ldots \\
14 \\
15 \\
16 \\
\end{array}$ & $\begin{array}{c}457.34 \\
\ldots . \\
487.87 \\
498.04 \\
508.22 \\
\end{array}$ \\
\hline $\begin{array}{c}3 \\
(\mathrm{MS}-2)\end{array}$ & $525-605$ & 567.2 & $\begin{array}{l}18 \\
\ldots \\
22 \\
23 \\
\ldots \\
25\end{array}$ & $\begin{array}{c}528.57 \\
\ldots \\
569.27 \\
579.45 \\
\ldots \\
599.80\end{array}$ \\
\hline $\begin{array}{c}4 \\
(\mathrm{MS}-3)\end{array}$ & $630-690$ & 660 & $\begin{array}{l}28 \\
\ldots \ldots \\
\mathbf{3 1} \\
\ldots \\
33\end{array}$ & $\begin{array}{c}630.32 \\
\ldots \\
660.85 \\
\ldots \\
681.200 \\
\end{array}$ \\
\hline $\begin{array}{c}5 \\
(\mathrm{MS}-4)\end{array}$ & $775-805$ & 790 & $\begin{array}{l}42 \\
43 \\
44 \\
45\end{array}$ & $\begin{array}{l}772.78 \\
782.95 \\
793.13 \\
803.30 \\
\end{array}$ \\
\hline$\left(\begin{array}{c}6 \\
\left(\mathrm{MS}-4^{\prime}\right)\end{array}\right.$ & $845-890$ & 865.6 & $\begin{array}{l}49 \\
50 \\
51 \\
\ldots 3 \\
5\end{array}$ & $\begin{array}{c}844.00 \\
854.18 \\
864.35 \\
\ldots \\
884.70 \\
\end{array}$ \\
\hline $\begin{array}{c}7 \\
(\text { MS-5') }\end{array}$ & $1200-1300$ & 1244.4 & $\begin{array}{c}\frac{106}{\ldots} \\
\ldots 10 \\
\ldots \\
115\end{array}$ & $\begin{array}{c}1205.07 \\
\ldots \\
1245.36 \\
\ldots \\
1295.86 \\
\end{array}$ \\
\hline $\begin{array}{c}8 \\
\text { (MS-5) }\end{array}$ & $1550-1750$ & 1640.1 & $\begin{array}{c}141 \\
\ldots \\
149 \\
\ldots \\
160 \\
\end{array}$ & $\begin{array}{c}1558.12 \\
\ldots \\
1638.81 \\
\ldots \\
1749.70\end{array}$ \\
\hline$\stackrel{9}{(\mathrm{MS}-7)}$ & $2080-2350$ & 2225.7 & $\begin{array}{c}195 \\
\ldots \\
202 \\
204 \\
\ldots \\
207 \\
208 \\
211\end{array}$ & $\begin{array}{c}2102.94 \\
\ldots \\
2173.53 \\
2193.73 \\
\ldots \\
2224.03 \\
2234.12 \\
2264.32 \\
\end{array}$ \\
\hline
\end{tabular}

first we obtained the transformation and rotation parameters using the algorithm mentioned in [10], which is based on optimization of mutual information between two images. First we applied the obtained transformations and rotations on the Hyperion data to obtain a registered Hyperion to ALI image. Then, we refined the registration using ENVI 3.5 [11] using nearest neighbor linear interpolation and by manual selection of ground control points. Then, we chose a subset of size $384 \times 128$ from both ALI and Hyperion data sets. Thus, we have 242 bands of Hyperion and 9 bands of ALI, all from the same location and of the same size. 


\section{APPROACH}

In this section we go over three image fusion techniques we used in our study of fusion of ALI and Hyperion. Two well known methods for image fusion are PCA and wavelet-based fusion. We also go over details of cokriging as an interpolation method and propose using it for image fusion.

\section{A. $P C A$}

Principle Component Analysis (PCA) is a statistical technique that transforms a multivariate data set of inter-correlated variables into a data set of new uncorrelated linear combinations of the original variables [5]. There are two ways of using PCA for image fusion: one approach is concerned with fusion of one multispectral data with an image with higher spatial resolution. The second approach considers fusion of images with the same spatial resolution [5], [12]. In the latter approach, images are stacked together as one multispectral image, and PCA is performed on all of the concatenated data. We use the second approach of using PCA for image fusion in this paper.

\section{B. Wavelet-Based Fusion}

Briefly, a wavelet decomposition of any given signal (1-D or 2-D) is the process that provides a complete representation of the signal according to a well-chosen division of the timefrequency (1-D) or space-frequency (2-D) plane [13]. Through iterative filtering by low-and high-pass filters, it provides information about low- and high-frequencies of the signal at successive spatial scales. For fusion purposes, multi-resolution wavelet decomposition separates high- and low-frequency components of the two given data sets and these components are then recomposed differently in the reconstruction phase.

In our experiments, we are using a Daubechies filter [13] of size 2 and a Mallat Multi-Resolution Analysis (MRA) [14] decomposition and reconstruction scheme. Figure 1 illustrates the wavelet-based fusion idea, where high- and low-resolution data are independently decomposed using the MRA wavelet decomposition process. Then, components from both decompositions are combined during the reconstruction phase to create the new fused data. In this scheme and similar to [15], where different spatial resolution data are fused, we fuse the different spectral resolution data in the following manner: lowfrequency information of the lowest spectral resolution data (e.g., ALI data) is combined with high-frequency information of the highest spectral resolution data (e.g., Hyperion data). In our experiments, the same Daubechies filter size 2 is used for both decomposition and reconstruction phases and for both types of data.

\section{Cokriging}

We first give an overview of the cokriging interpolation method, and then propose utilizing this method for data fusion.
Fig. 1. Wavelet-Based Fusion

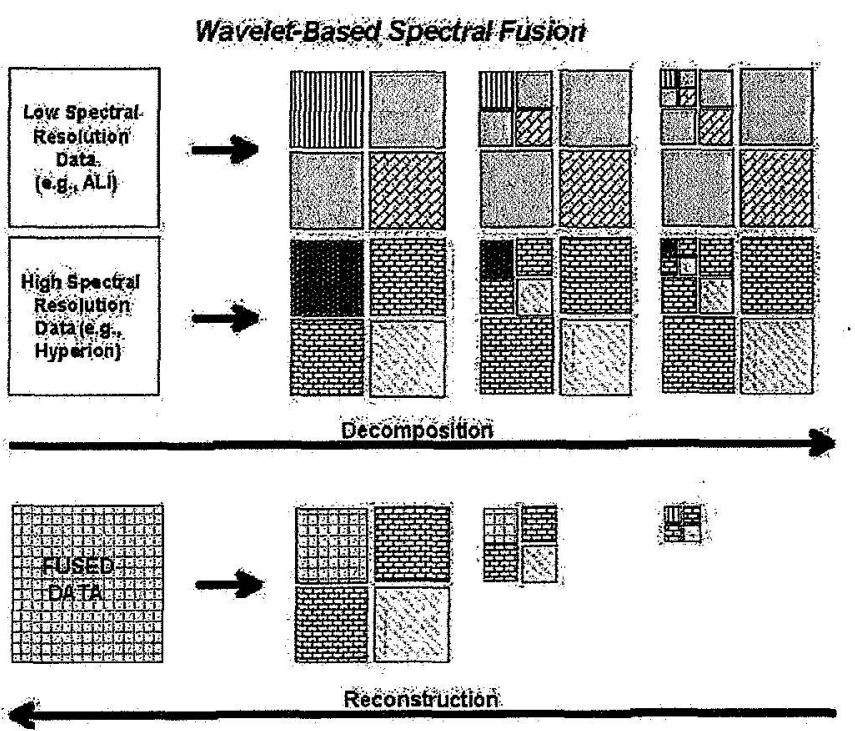

1) Cokriging as an Interpolation Method: Cokriging has been traditionally used in mining and geostatistics applications [2]-[4]. Cokriging is a method for estimation that minimizes the variance of the estimation error by taking into consideration the spatial correlation between the variables of interest and the secondary variables. In other words, a function $\mathrm{U}$ at location 0 is estimated as a linear combination of both the variable of interest and the secondary variable(s). That is, in the case where we have one secondary variable, the estimate of $U$ at location $0, \hat{u}_{0}$, using the two variables as mentioned in [4], is given by $\hat{u}_{0}=$ $\sum_{i=1}^{n} a_{i} u_{i}+\sum_{j=1}^{m} b_{j} v_{j}$, where $u_{1}, u_{2}, \ldots, u_{n}$ are primary data at $n$ nearby locations, $v_{1}, v_{2}, \ldots, v_{n}$ are secondary data at $m$ nearby locations, and $a_{1}, a_{2}, \ldots, a_{n}$ and $b_{1}, b_{2}, \ldots, b_{m}$ are cokriging weights which are needed to be found and calculated. The estimation error, $R$, is calculated as $R=$ $\widehat{U}_{0}-U_{0}=w^{t} Z$; where $w^{t}=\left(a_{1}, \ldots, a_{n}, b_{1}, \ldots, b_{m},-1\right)$, and $Z^{t}=\left(U_{1}, \ldots, U_{i}, V_{1}, \ldots, V_{m}, U_{0}\right)$. The goal of cokriging is to find the weight vector $w^{t}$ such that the variance of the error is minimized and the estimate for $\hat{U}_{0}$ be unbiased, that is, the mean error residual is zero.

- There are various types of cokriging methods. The distinction arises from the way in which constraints are imposed. Three common types of cokrigingA are: ordinary, simple, and standardized cokriging (see [3], p. 204, and [4], ch. 17). Here we illustrate the ordinary cokriging. Ordinary cokriging requires that $\sum_{i=1}^{n} a_{i}=1$ and $\sum_{j=1}^{m} b_{j}=0$ in the above equation. These two constraints are there to make our estimate unbiased, or to minimize the variance of our estimation error. 
From the definition of variance, we have

$$
\begin{aligned}
\operatorname{Var}(R) & =w^{t} C_{Z} w \\
& =\sum_{i}^{n} \sum_{j}^{n} a_{i} a_{j} \operatorname{Cov}\left(U_{i} U_{j}\right) \\
& +\sum_{i}^{m} \sum_{j}^{m} b_{i} b_{j} \operatorname{Cov}\left(V_{i} V_{j}\right) \\
& +2 \sum_{i}^{n} \sum_{j}^{m} \operatorname{Cov}\left(U_{i} V_{j}\right)-2 \sum_{i}^{n} a_{i} \operatorname{Cov}\left(U_{i} U_{0}\right) \\
& -2 \sum_{j}^{m} b_{j} \operatorname{Cov}\left(V_{j} U_{0}\right)+\operatorname{Cov}\left(U_{0} U_{0}\right) .
\end{aligned}
$$

We leave the proof of why the two mentioned constraints ensure unbiasedness of our estimate as an exercise for the reader. So now we have an optimization problem with two constraints. This is where we take advantage of Lagrange multipliers [16]. Let our Lagrange multipliers be $\mu_{1}$ and $\mu_{2}$. Then, we are trying minimize $\operatorname{Var}(R)$ subject to the two mentioned constraints by solving for coefficients $a_{1} \ldots a_{n}, b_{1} \ldots b_{m}, \mu_{1}, \mu 2$, where

$$
\operatorname{Var}(R)=w^{t} C_{Z} w+2 \mu_{1}\left(\sum_{i=1}^{n} a_{i}-1\right)+2 \mu_{2}\left(\sum_{j=1}^{m} b_{j}\right) \text {. }
$$

The next step is taking partial derivatives of the above equation with respect to all $n+m$ cokriging variables and the two Lagrange multipliers and setting them to zero. Then, we have the following $n+m+2$ equations to solve:

$$
\begin{gathered}
\sum_{i=1}^{n} a_{i} \operatorname{Cov}\left(U_{i} U_{j}\right)+\sum_{i=1}^{m} b_{i} \operatorname{Cov}\left(V_{i} U_{j}\right)+\mu_{1}=\operatorname{Cov}\left(U_{0} U_{j}\right) \\
\text { for }(j=1 \ldots n), \\
\sum_{i=1}^{n} a_{i} \operatorname{Cov}\left(U_{i} V_{j}\right)+\sum_{i=1}^{m} b_{i} \operatorname{Cov}\left(V_{i} V_{j}\right)+\mu_{2}=\operatorname{Cov}\left(U_{0} V_{j}\right) \\
\text { for }(j=1 \ldots m), \\
\sum_{i=1}^{n} a_{i}=1, \text { and } \sum_{i=1}^{m} b_{i}=0 .
\end{gathered}
$$

Once the above system of equations is solved, we have the necessary coefficients $a_{1}, a_{2}, \ldots, a_{n}, b_{1}, b_{2}, \ldots, b_{m}$ to estimate function $U$ at location 0 . Note that the above mentioned method works only for point estimation.

Instead of having one set of secondary variables $V_{1} \ldots V_{m}$, we may use multiple sets of secondary variables. Each additional set of secondary variables $W_{1} \ldots W_{k}$ will introduce a new set of coefficients $c_{1} \ldots c_{k}$ and a new lagrange multiplier $\mu_{w}$.

For the general case where we have $s$ set of variables (as oppose to just 2 sets, one primary and one secondary), our linear system will be as follows:

$$
\left(\begin{array}{cc}
C & E \\
E^{T} & 0
\end{array}\right)\left(\begin{array}{c}
T \\
\mu
\end{array}\right)=\left(\begin{array}{c}
C 0 \\
I 0
\end{array}\right)
$$

, where $C$ is the covariance (or its estimate) matrix of all known variables' pair, and $C_{0}$ is the vector of pairwise covariances between the unknown variable $U_{0}$ and all other known variables.

$\mu$ is the vector of all lagrange multipliers $\mu_{1} \ldots \mu_{s} . E$ is a vector of matrices $I_{1} \ldots I_{s}$. Each matrix $I_{i}, i \in\{1 \ldots s\}$ is of size (number of points in $i^{t h}$ variable set) $\times s$. All elements in the $i^{\text {th }}$ column of $I_{i}$ are one and all other entries are zero.

$T$ is the vector of all coefficients, and $I_{0}$ is a column vector of of size $s \times 1$ of all elements under $C_{0}$ on the right hand side of the equation. Similarly to ensure unbiasedness, this vector is made of a 1 on top and all zeros for the rest of entries. It can also be proven that in order for the above system to have a solution, we need $C$ to be positive definite.

2) Cokriging as a Fusion Method: While PCA and wavelet-based fusion have been traditionally used for image fusion, they have their own shortcomings. For PCA most information is gained if all calibrated and visually good quality multispectral input bands are used (rather than a selective subset). PCA results are also very sensitive to the selected area for fusion [5]. Also, PCA is a general purpose approach and no application-oriented information is used for PCA. Wavelet based fusion can deal with images of different spectral and spatial resolutions. However, this method cannot handle cases where data is scattered and rather sparse (either in spatial or spectral dimension), or when input images differ greatly in either their spectral or spatial resolutions.

Thus, we think using cokriging can help us integrate data of various sources with different spatial and spectral resolutions according to the application in mind. Our image/data fusion problem is then considered as an interpolation problem where we want to estimate frequencies at missing data points for data with low spatial resolution, or re-estimate or come up with better frequencies for data points with lower spectral resolution. That is, we can perform cokriging as a fusion method either in spatial or spectral domain.

In spatial domain, location values for our interpolation problem are either the geographic coordinates of data points or their pixel coordinates in high spatial resolution image. We will have two sets of variables in our problem to deal with: data with high spatial resolution and data with low spatial resolution. The first variable will have a value at almost all specified locations while the second variable will have missing values since it has lower spatial resolution. Our goal will be to come up with the missing values for the second variable through cokriging. That is we are increasing the spatial resolution of the image with lower spatial resolution.

Similarly, when performing fusion in spectral domain, we have two sets of variables. One is our image with high spectral resolution and the other is the image with low spectral resolution which we want to improve. In this case, center of wavelength intervals of each data set serves as the location/coordinate values. Each band of data with high spectral resolution will have a reflectance value at each interval while the low spectral data will have some missing values. 


\section{EXPERIMENTS}

In this section we go over details of the experiments we performed using three image fusion techniques for our study of fusion of ALI and Hyperion.

\section{A. PCA}

We performed fusion of ALI and Hyperion data using the PCA approach to see how the classification of the fused data would change with respect to classification of data when using only either the ALI or Hyperion data. To do so we performed 3 series of PCA transformations on three sets of data:

- 9 bands of ALI data.

- Calibrated bands of Hyperion which do not appear corrupted visually (total of 140 bands as shown in Table I).

- All ALI bands and Hyperion bands mentioned above stacked together as one multi-spectral data set (we did not have to worry about their spatial resolutions since they all share the same spatial resolution). Thus, we had a data set with 149 bands.

From each case's resulted PCs, the first set of PCs is chosen is such a way that it contains at least $99 \%$ of the original data sets' information content (defined as ratio of the sum of their eigenvalues to the sum of all PCs eigenvalues).

\section{B. Wavelet-Based Fusion}

TABLE II

RECOMMENDED HYPERION BANDS TO FUSE WITH EACH ALI BAND BASED ON CORRELATION VALUES, (C)

\begin{tabular}{|c|c|c|}
\hline $\begin{array}{c}\text { ALI } \\
\text { MS Bands }\end{array}$ & $\begin{array}{c}\text { Matching Hyp } \\
\text { Band w/ Least C }\end{array}$ & $\begin{array}{c}\text { C } \\
\text { Value }\end{array}$ \\
\hline 1 (MS-1') & 10 & 0.861991 \\
2 (MS-1) & 15 & 0.859726 \\
3 (MS-2) & 25 & 0.843684 \\
4 (MS-3) & 33 & 0.83889 \\
5 (MS-4) & 45 & 0.782766 \\
6 (MS-4') & 53 & 0.773323 \\
7 (MS-5') & 113 & 0.772634 \\
8 (MS-5) & 160 & 0.708357 \\
9 (MS-7) & 198 & 0.16961 \\
\hline
\end{tabular}

For wavelet based fusion we fused each band of ALI data is fused with one chosen Hyperion band whose wavelength range lies within that ALI band's wavelength range. That is each ALI band can be fused with one of many corresponding Hyperion bands. We performed two sets of wavelet based fusions based on how we chose the Hyperion band to be fused with each ALI band:

- In the first experiment, we chose Hyperion bands to be fused with ALI based on our target application. That is, from candidate Hyperion bands we selected the one among those whose wavelength shows a typical vegetation reflectance in general, and a reflectance of vegetation types of interest to ISFS in particular, has the highest reflectance [7]. The 9 chosen Hyperion bands for fusion with 9 bands of ALI are underlined in Table I.
- In the second experiment, we choose bands that yields inputs with the highest variations, regardless of the application. That is from the list of Hyperion candidate bands for fusion with each ALI band, we chose the Hyperion band which is the least correlated to the corresponding ALI band. The chosen Hyperion bands for this experiment with their correlation values are shown in Table II.

\section{Cokriging}

Since both multispectral ALI and Hyperion data have the same spatial resolution, we can only perform fusion on the spectral dimension. That is, we can improve spectral quality of ALI by cokriging it with both ALI and Hyperion data. Looking at Table I, we can see there are some wavelength ranges which are not covered by ALI data. In particular, considering only calibrated bands of Hyperion which did not seem visually corrupted, wavelengths covered by Hyperion bands 17, 26$27,34-41,46-48,54-105,116-140,161-194$, and bands 220 224 are not covered by ALI bands. Thus, one could create 8 new bands of ALI through cokriging, where each new ALI band will cover the missing intervals of the spectrum. Another fusion goal might also be to interpolate ALI only at a particular wavelength of interest, based on application.

Fig. 2. ALI and Hyperion Reflectance in Their Spectral Domain

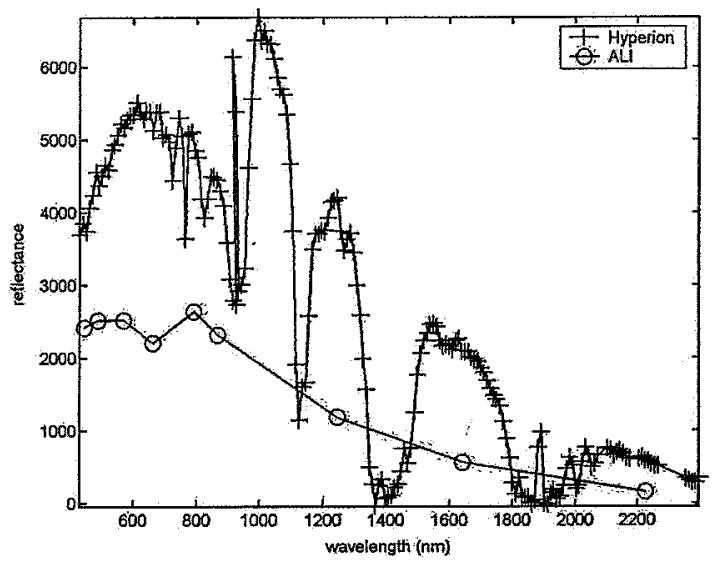

Figure 2 shows the reflectance at one pixel both with the Hyperion and ALI sensors. Our experiments deal with estimating ALI values at missing intervals by using both $\mathrm{ALI}$ and Hyperion, and investigate how we can mimic Hyperion's trend at wavelengths of interest for ALI. This is done by by first interpolating $\mathrm{ALI}$ at one wavelength location at each interval by estimating ALI values at wavelengths matching centers of wavelength ranges for Hyperion bands 17, 26, 37, 47, 77, 134, and 180. In the second experiment, we get a smoother ALI coverage, by estimating ALI at wavelength centers of Hyperion bands $17,26,37,39,47,57,77,97,130,134,138,170,180$, and 190. Finally, we examine how cokriging would perform if we were to reconstruct ALI at every single interval where we have Hyperion coverage. This demonstrates how cokriging 
performs for the spectral fusion of ALI with Hyperion. In practice, a user could specify the intervals in which he/she is interested to have ALI coverage based on the application, and thus only a few new/fused bands will be constructed for ALI through this process.

\section{Evaluation Methods}

Classification of fusion results and comparison with classifications obtained by using either ALI or Hyperion data is the ideal way of evaluating our fusion results for our ISFS project purposes. However, this would require ground truth data which is not yet available, although it should soon be acquired from field measurements. Thus, for this study, we use other spectral quality metrics.

While there are a few fusion quality metrics introduced by [17], [18], they present some limitations in our experimental framework. In particular, the previous metrics only deal with data of gray scale with values between 0-255. Additionally, these measures do not handle multi-spectral data.

Since texture is one of the main characteristics usually used for classifying images [19], [20], we propose to utilize texture-related measurements as our fusion quality metrics. Haralick [19] first proposed using a co-occurrence matrix to calculate various statistical texture properties for an image. A co-occurrence matrix calculates the number of occurrences of all pairs of gray level which are separated by a distance $d$ along a given direction. From the co-occurrence matrix, several texture measurements can be computed among which are variance and entropy. For evaluation of our waveletbased fusion results, we propose to use the variance of cooccurrence matrices as a fusion quality metric. That is, for each input and fused image, we calculate a co-occurrence variance image based on variances computed from local co-occurrence matrices [19], [20]. Since a higher mean image variance is associated with a higher amount of texture, an image with a higher mean of its variance image will also probably result in a more accurate classification. Having more texture in our fusion result will indicate that some information was gained from each of the input images. While using statistical texture measures will tell us how different each fused result is compared to input bands, we also need to ensure that our fused result contains information from each input band as well. Thus, for our wavelet based fusion, another spectral quality metric is considered: correlation. We calculate correlation of each fused band to each of their input bands. The idea is that each fused band need to be highly correlated with its input bands while having more texture associated with it.

\section{RESULTS}

This section summarizes the fusion results obtained from our PCA, wavelet-based fusion, and cokriging experiments. For PCA and wavelet-based fusion experiments, as a qualitative validation of the fusion methods, we first performed clustering using $\mathrm{k}$-means algorithm with $k=7$ and a maximum of 15 iterations on both results and input. Then, we evaluated the clustering results by calculating statistical texture measures as
TABLE III

MEAN OF VARIANCE IMAGES OF ALI, HYPERION, AND FUSED BANDS IN FUSION THROUGH PCA, $V=$ MEAN OF VARIANCE IMAGE

\begin{tabular}{|c|c|c|}
\hline ALI V & Hyp V & Fused V \\
\hline 143.98 & 137.64 & 180.10 \\
\hline
\end{tabular}

proposed by Haralick and mentioned in Section VII. For the cokriging experiment we show preliminary results of fusion performed on one pixel in the spectral domain.

For PCA, we performed clustering on the first set of PCs that contained more than $99 \%$ of the original multispectral image information, that is, the first 3 PCs of ALI data, the first 7 PCs of Hyperion data, and the first 9 PCs of fused data. Results are shown in Figure 3. When using PCA, only the overall mean of variance images can be calculated. Table III shows that this overall variance of the PCA-fused image has increased compared to the PCA results obtained only from ALI or Hyperion data.

Fig. 3. Classifications of PCA Results of ALI and Hyperion Images Separately and Classification of Their PCA Based Fusion Results

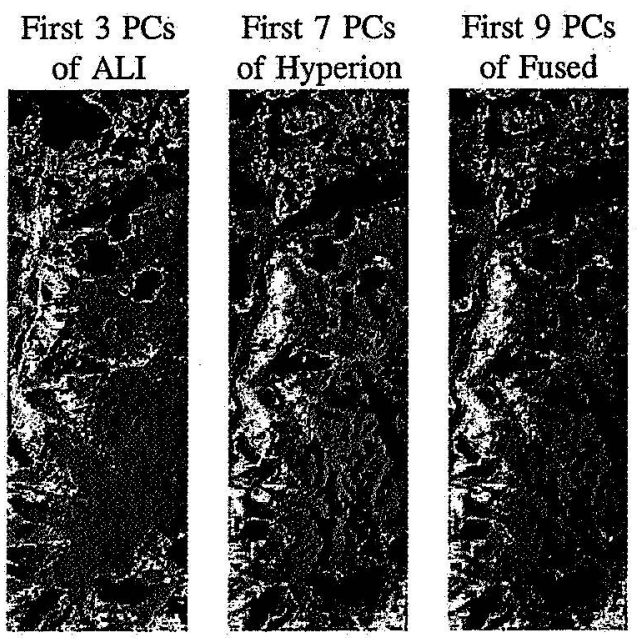

Figures 4 and 5 show k-means classifications performed on all 9 bands of ALI, the chosen 9 bands of Hyperion in both wavelet based fusion experiments, and the 9 bands of fused results respectively. Tables IV and VI show mean values of variance images calculated as pointed out in Section VII. We see that in both experiments, the first 7 fused bands have higher variance than each of their associated ALI and Hyperion input bands. We also see in Table V, that the first 7 fused bands in both experiments are highly correlated with their associated Hyperion band. Thus, while the spectral quality of high spectral resolution data is saved in fused bands, fused bands have more texture associated with them. In both experiments, band 8 of fused data has more variance than its input Hyperion band but less that its associated ALI band. This is partly due to the fact that ALI band 8 is rather noisy and corrupted. In both cases no improvement was gained in fused 
Fig. 4. Classifications of Input ALI and Hyperion Images to Wavelet Based Fusion Experiment 1 and Its Result
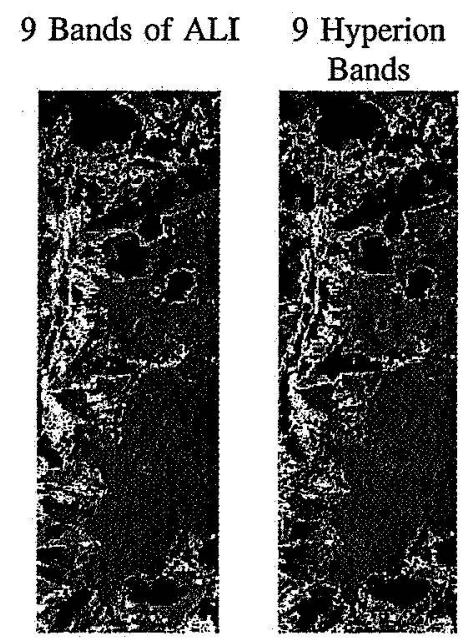

9 Fused Bands

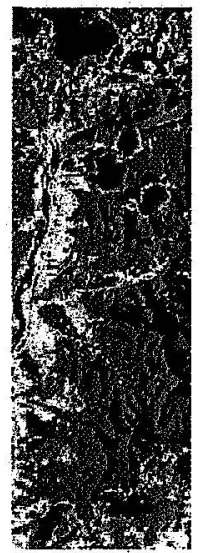

Fig. 5. Classifications of Input ALI and Hyperion Images to Wavelet Based Fusion Experiment 2 and Its Result
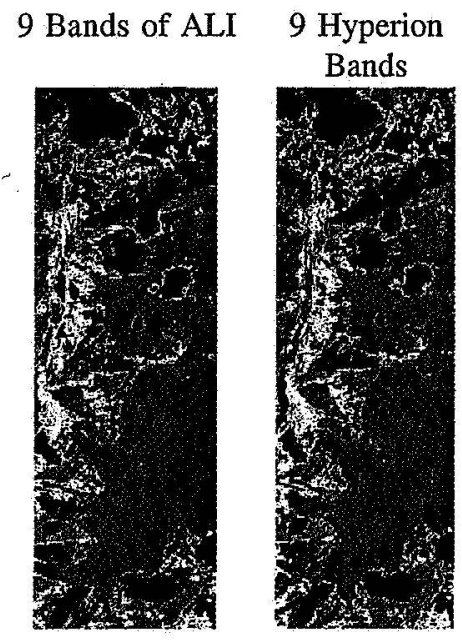

9 Fused Bands

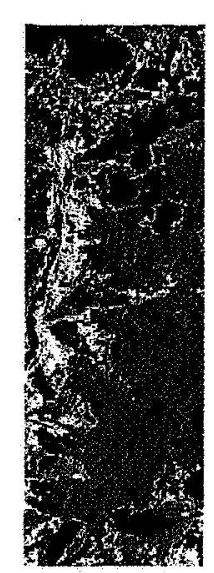

band 9. In the first experiment, there is a greater increase in variance images of the fused results than that of experiment 2 . This is why we see more detailed clustering results in Figure 4 than in Figure 5. The overall varinace quantities confirm this since in the second experiment we see overall improvement over Hyperion data only and not over ALI. In general, waveletbased fusion works better on the first 7 bands. However, this method can be used to reduce the effect of noisy data in ALI data in bands 8 and 9.

In our preliminary results for the cokriging, Figures 6,7 , and 8 show that as we increase the number of wavelengths at which the ALI data is interpolated, we can construct ALI bands which mimic Hyperion's performance while incorporating ALI's values. Of course, one will choose intervals of interest to perform this cokriging so that instead of dealing with 242
TABLE IV

MEAN OF VARIANCE IMAGES OF ALI, HYPERION, AND FUSED BANDS IN WAVELET BASED FUSION, EXPERIMENT 1

\begin{tabular}{|c|c|c|c|c|c|}
\hline ALI & $\mathrm{V}$ & Hyp & $\mathrm{V}$ & Fused & $\mathrm{V}$ \\
\hline$A_{1}$ & 77.62 & $H_{9}$ & 85.63 & $F_{1}$ & 113.84 \\
$A_{2}$ & 99.49 & $H_{16}$ & 116.96 & $F_{2}$ & 138.72 \\
$A_{3}$ & 139.51 & $H_{23}$ & 158.87 & $F_{3}$ & 183.63 \\
$A_{4}$ & 193.03 & $H_{28}$ & 192.84 & $F_{4}$ & 212.16 \\
$A_{5}$ & 169.97 & $H_{43}$ & 176.24 & $F_{5}$ & 200.32 \\
$A_{6}$ & 168.54 & $H_{50}$ & 180.82 & $F_{6}$ & 208.95 \\
$A_{7}$ & 164.22 & $H_{106}$ & 157.81 & $F_{7}$ & 197.47 \\
$A_{8}$ & 344.53 & $H_{160}$ & 190.52 & $F_{8}$ & 261.85 \\
$A_{9}$ & 260.68 & $H_{195}$ & 179.91 & $F_{9}$ & 240.52 \\
\hline Overall & & & & & \\
$\mathrm{V}$ & 179.73 & & 159.96 & & 195.27 \\
\hline
\end{tabular}

TABLE V

CORRELATION OF FUSED DATA WITH High SPECTRAL RESOLUTION INPUT FOR WAVELET-BASED FUSION, EXPERIMENT 1 AND 2

\begin{tabular}{|c|c|c|c|}
\hline $\begin{array}{c}\text { Exp1: Hyperion } \\
\text { Fused Pairs }\end{array}$ & $\mathrm{C}$ & $\begin{array}{c}\text { Exp2: Hyperion } \\
\text { Fused Pairs }\end{array}$ & $\mathrm{C}$ \\
\hline$H_{9}, F_{1}$ & 0.929 & $H_{10}, F_{1}$ & 0.956 \\
$H_{16}, F_{2}$ & 0.949 & $H_{15}, F_{2}$ & 0.965 \\
$H_{23}, F_{3}$ & 0.955 & $H_{25}, F_{3}$ & 0.972 \\
$H_{28}, F_{4}$ & 0.952 & $H_{33}, F_{4}$ & 0.969 \\
$H_{43}, F_{5}$ & 0.913 & $H_{45}, F_{5}$ & 0.934 \\
$H_{50}, F_{6}$ & 0.890 & $H_{53}, F_{6}$ & 0.914 \\
$H_{106}, F_{7}$ & 0.873 & $H_{113}, F_{7}$ & 0.901 \\
$H_{160}, F_{8}$ & 0.592 & $H_{160}, F_{8}$ & 0.679 \\
$H_{195}, F_{9}$ & 0.385 & $H_{198}, F_{9}$ & 0.826 \\
\hline
\end{tabular}

bands of Hyperion, or only 9 bands of ALI, one can get a full spectrum coverage through about 17 ALI bands ( 9 original and about 8 or more fused ALI bands).

\section{CONCLUSiOn AND FUtURE WORK}

Experiments for the fusion of ALI and Hyperion data using PCA, wavelet-based fusion, and cokriging have been performed. Fusion results based on PCA and wavelets show that texture, measured through variance, can be improved through fusion, while preserving almost all the input original information. Variance and correlation measurements were utilized to validate the results and form the basis for a new

TABLE VI

MEAN OF VARIANCE IMAGES OF ALI, HYPERION, AND FUSED BANDS IN WAVELET BASED FUSION, EXPERIMENT 2

\begin{tabular}{|c|c|c|c|c|c|}
\hline ALI & $\mathrm{V}$ & Hyp & $\mathrm{V}$ & Fused & $\mathrm{V}$ \\
\hline$A_{1}$ & 77.62 & $H_{10}$ & 90.14 & $F_{1}$ & 101.65 \\
$A_{2}$ & 99.49 & $H_{15}$ & 111.12 & $F_{2}$ & 118.27 \\
$A_{3}$ & 139.51 & $H_{25}$ & 174.84 & $F_{3}$ & 176.30 \\
$A_{4}$ & 193.03 & $H_{33}$ & 217.32 & $F_{4}$ & 225.68 \\
$A_{5}$ & 169.97 & $H_{45}$ & 169.88 & $F_{5}$ & 182.35 \\
$A_{6}$ & 168.54 & $H_{53}$ & 166.87 & $F_{6}$ & 184.36 \\
$A_{7}$ & 164.22 & $H_{113}$ & 182.49 & $F_{7}$ & 197.00 \\
$A_{8}$ & 344.53 & $H_{160}$ & 190.52 & $F_{8}$ & 205.10 \\
$A_{9}$ & 260.68 & $H_{198}$ & 184.84 & $F_{9}$ & 173.18 \\
\hline Overall & & & & & \\
V & 179.73 & & 165.34 & & 173.77 \\
\hline
\end{tabular}


